\title{
Comprendiendo la conducta del paciente con diabetes: estrategias para mejorar la adherencia terapéutica
}

\section{Understanding the behavior of patients with diabetes: strategies to improve therapeutic adherence}

\author{
Jorge Villalba-Arias $\mathbb{1}^{1,2}$, José Almirón-Santacruz $\mathbb{1}^{1}$, Julio Torales $\mathbb{1}^{1,3}$
}

${ }^{1}$ Universidad Nacional de Asunción, Facultad de Ciencias Médicas, Cátedra de Psiquiatría, San Lorenzo, Paraguay.

Universidad Nacional de Asunción, Facultad de Ciencias Médicas, Cátedra de Psiquiatría (Filial Santa Rosa), Santa Rosa del Aguaray,

Paraguay.

${ }^{3}$ Universidad Nacional de Asunción, Facultad de Ciencias Médicas, Cátedra de Socioantropología (Filial Santa Rosa), Santa Rosa del Aguaray, Paraguay.

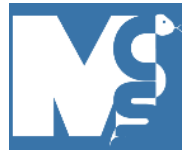

Recibido: 08/02/2021

Revisado: 20/02/2021

Aceptado: 24/02/2021

\section{Autor correspondiente}

Julio Torales

Universidad Nacional de Asunción, Paraguay

juliotorales@gmail.com

\section{Conflictos de interés}

Los autores declaran no poseer conflictos de interés.

\section{Fuente de financiación}

Los autores no recibieron apoyo financiero para la investigación, autoría y/o publicación de este artículo.

Este artículo es publicado bajo una licencia de Creative Commons Reconocimiento 4.0 Internacional.

\section{RESUMEN}

La diabetes es una enfermedad crónica que, si no es tratada adecuadamente, se acompaña de muchas comorbilidades, complicaciones, pobre calidad de vida, así como la posibilidad de un desenlace fatal. La falta de adherencia terapéutica es una barrera a vencer para mejorar la calidad de vida y la salud de los pacientes afectados. En este artículo se hace un repaso narrativo sobre los conceptos en los que se funda la adherencia terapéutica, explicando los modelos sobre los que sostiene el duelo ante una enfermedad crónica o terminal, resumiendo los conceptos que hacen a la importancia de la comunicación, a la construcción de conductas terapéuticas, a la motivación-acción y a las emociones en salud, aristas todas que forman parte del constructo conocido como "adherencia terapéutica". Por último, se presentan recomendaciones sucintas para mejorar esa adherencia, involucrando tanto al paciente como al médico en su desarrollo, con el fin de incrementar el éxito terapéutico.

Palabras clave: diabetes; adherencia terapéutica; relación médico-paciente; calidad de vida.

\section{ABSTRACT}

Diabetes is a chronic disease that, if not treated properly, is accompanied by many comorbidities, complications, poor quality of life, as well as the possibility of a fatal outcome. Lack of therapeutic adherence is a barrier to overcome in order to improve the quality of life and health of affected patients. In this article a narrative review is made of the concepts on which therapeutic adherence is based, explaining the models on which grief sustains in the face of a chronic or terminal illness, summarizing the concepts that make the importance of communication, of the construction of therapeutic behaviors, of motivation-action and of emotions in health, all of which are part of the construct known as "therapeutic adherence". Finally, succinct recommendations are presented to improve adherence, involving both the patient and the physician in its development, in order to increase therapeutic success.

Keywords: diabetes; therapeutic adherence; doctor-patient relationship; quality of li. 


\section{INTRODUCCIÓN}

La diabetes es un cuadro que presenta dos componentes que juegan en contra de un buen tratamiento: por un lado, es una enfermedad crónica, lo cual implica un tratamiento que debe acompañarse de cambios en el estilo de vida del paciente $y$, por otro lado, es una enfermedad que durante años se muestra silente (aun cuando existan hallazgos laboratoriales que indiquen el diagnóstico, por lo que es probable que transcurran años antes de la aparición de síntomas e incluso otros signos) $(1,2)$.

Si el objetivo del lector es simplemente el de obtener las estrategias para mejorar la adherencia terapéutica, todas ellas pueden ser resumidas en dos recomendaciones: mejorar la relación médicopaciente y pasar, de una vez por todas, del Modelo Biomédico al Modelo Biopsicosocial (3). Desarrollar estas dos ideas es lo que se hará de aquí en adelante, intentando (a través de este artículo de revisión) comprender la renuencia de los pacientes con diabetes a seguir y cumplir con las indicaciones dadas por el personal de blanco.

\section{DESARROLLO}

De los modelos explicativos a una explicación teórica del duelo

Desde que George Engel desarrollase el Modelo Biopsicosocial a mediados de los 50 del siglo pasado (e invitase con ello a abandonar el Modelo Biomédico) han pasado más de 60 años. Pero, si bien los programas académicos han sido actualizados para este nuevo enfoque, lamentablemente la práctica dista aún de ello. Existen diferencias cardinales entre un modelo y otro que juegan un papel fundamental a la hora de comprender a la enfermedad y el rol del enfermo. Por ejemplo, en el Modelo Biomédico el concepto de salud requiere meramente la ausencia de enfermedades, mientras que en el modelo biopsicosocial se habla de un bienestar biológico, psicológico y social como criterio necesario para la salud $(4,5)$.

Otra diferencia importante entre el Modelo Biopsicosocial y el Biomédico es que este último centra la mecánica de la enfermedad solamente en el paciente mientras que el modelo biopsicosocial amplía los componentes hacia el ambiente del paciente. Entonces, desde la perspectiva de este nuevo paradigma, no es lo mismo padecer disentería en un país de bajos ingresos que en uno de altos. En otras palabras, desde el Modelo Biopsicosocial no sólo las características genéticas del paciente juegan un papel preponderante en el pronóstico de este, también lo hacen sus habilidades psicológicas y su respaldo familiar-social $(4,5)$.

Pasaron varios años desde que Elisabeth Kübler-Ross presentase (en 1969), sus cinco etapas del duelo basándose en las observaciones que esta médica psiquiatra realizó sobre la conducta de pacientes con cuadros terminales y en cuidados paliativos. Tales etapas (junto con sus descripciones) están resumidas en la tabla $1(6,7)$.

TABLA 1. ETAPAS DEL DUELO SEGÚN EL MODELO KÜBLER-ROSS $(6,7)$.

\begin{tabular}{|c|c|c|}
\hline Etapa & Expresiones comunes del paciente & Características \\
\hline Negación & $\begin{array}{l}\text { "Yo estoy bien, me siento bien" } \\
\text { "Esto no me puede estar pasando" }\end{array}$ & $\begin{array}{l}\text { La negación es un mecanismo de defensa temporal utilizado por } \\
\text { el individuo. La negación es generalmente reemplazada luego } \\
\text { con una sensibilidad aumentada a las situaciones. }\end{array}$ \\
\hline Ira & $\begin{array}{c}\text { “Por qué a mí? iEsto es injusto" } \\
\text { "¿Cómo esto me puede estar } \\
\text { pasando a mí?” }\end{array}$ & $\begin{array}{l}\text { En esta segunda etapa, el individuo se da cuenta de que la } \\
\text { negación no puede continuar. En esta etapa es difícil cuidar a la } \\
\text { persona, ya que la misma siente ira y envidia. Cualquier persona } \\
\text { que simbolice la vida o la energía está expuesta al } \\
\text { resentimiento y la envidia proyectados por el paciente. }\end{array}$ \\
\hline Negociación & $\begin{array}{l}\text { "Señor mío, mi Dios, permíteme } \\
\text { vivir un poco más, para ver a mis } \\
\text { hijos graduarse" } \\
\text { "Haré cualquier cosa tener la } \\
\text { posibilidad de vivir un poco más" }\end{array}$ & $\begin{array}{l}\text { La tercera etapa implica la esperanza de que el individuo pueda } \\
\text { de alguna manera posponer o retrasar la muerte. Las } \\
\text { "negociaciones" para una vida más larga generalmente se } \\
\text { llevan a cabo con una deidad poder superior a cambio de una } \\
\text { forma de vida reformada. }\end{array}$ \\
\hline Depresión & $\begin{array}{l}\text { "Estoy muy triste, no vale la pena } \\
\text { hacer nada" } \\
\text { "Voy a morir, ¿qué sentido tiene?" } \\
\text { "Extraño a mi familia y amigos... no } \\
\text { vale la pena seguir" }\end{array}$ & $\begin{array}{l}\text { En la cuarta fase, el moribundo comienza a comprender la } \\
\text { seguridad de la muerte. Debido a esto, la persona puede } \\
\text { guardar silencio, rechazar las visitas y pasar mucho tiempo } \\
\text { llorando y lamentándose. Este proceso permite a la persona } \\
\text { moribunda romper con todos los sentimientos de amor y } \\
\text { cuidado. No es recomendable animar a una persona que se } \\
\text { encuentra en esta etapa. Es un momento importante que debe } \\
\text { procesarse. }\end{array}$ \\
\hline
\end{tabular}


"Esto debe pasar, ya no hay

Aceptación solución... debo prepararme para lo que se viene"
La etapa final llega con la paz y la comprensión de que la muerte se acerca. Generalmente, la persona quiere que la dejen sola. Las emociones y el dolor físico también pueden desaparecer. Esta fase también ha sido descrita como el final de la lucha contra la muerte.
Estudios posteriores y basados en las observaciones de la Dra. Kübler-Ross demostrarían que estas etapas no se dan necesariamente en el orden planteado por ella (y plasmado en la tabla 1), así como también se demostraría que estas etapas del duelo no se manifiestan solamente ante la inminencia de la muerte, sino también en el contexto de noticias traumáticas (por ejemplo, un divorcio).

Las enfermedades crónicas se caracterizan por varios factores, pero dos de ellos son particularmente importantes: su duración superior a los 6 meses y que su aparición implica necesariamente el cambio del estilo de vida del paciente. Estas dos características justifican categorizar la noticia de padecer de una enfermedad crónica como un evento con características de duelo. Esto último es uno de los principales motivos por los cuales los pacientes diagnosticados con diabetes (cualquiera de sus tipos), son renuentes a aceptar dicho diagnóstico (entre las estrategias que suelen adoptar estos pacientes al ser informados de este diagnóstico se encuentran la de pedir una segunda opinión profesional, la búsqueda de tratamientos "curativos" sin base científica, entre otros) (8).

\section{La importancia de la comunicación}

Antes de ingresar a los aspectos psicológicos que explican la falta de adherencia terapéutica, es importante fijarse en aspectos más básicos. Por ejemplo, aquellos que fueron determinados en el Consenso de Toronto en 1991 y se resumen en la tabla 2 (9).

TABLA 2. PROBLEMAS DETECTADOS POR EL CONSENSO DE TORONTO EN LA COMUNICACIÓN CLÍNICA (9).

- $\quad$ El $54 \%$ de las quejas de los pacientes y el $45 \%$ de sus preocupaciones no son comentadas con los profesionales.

- Hasta en un 50 \% de las consultas, los pacientes no estaban de acuerdo con su médico acerca de cuál era el problema principal de consulta.

- El tiempo medio del que disponen los pacientes para hablar sobre su problema antes de ser interrumpidos por los profesionales es de 18 segundos.

- La mayoría de las quejas de los pacientes respecto de sus médicos no hacen referencia a problemas de naturaleza técnica, sino a problemas de comunicación.

- Tanto los residentes como los médicos de planta muestran deficiencias severas en la comunicación cuando son evaluados.

- El lenguaje médico es, a menudo, técnico y confuso.

- Una proporción sorprendentemente elevada de los pacientes no entienden ni recuerdan lo que se les ha explicado sobre el diagnóstico o el tratamiento.

Como se puede apreciar, los diferentes puntos del Consenso de Toronto describen diferentes consecuencias de una deficiente comunicación. Es imposible esperar un comportamiento terapéutico adecuado del paciente si no comprende las indicaciones dadas. Por ello, es fundamental establecer estrategias que permitan determinar el su grado de comprensión. Por ejemplo, en lugar de preguntarle al paciente si entendió las instrucciones que le acabamos de dar, es preferible pedirle que nos explique lo que entendió de lo que le acabamos de explicar. Las preguntas cerradas/dicotómicas son aliadas del tiempo. Las preguntas abiertas son aliadas de la relación médico-paciente $y$, por tanto, de un mejor pronóstico basado en el cumplimiento terapéutico (9).
Es importante recordar que el tratamiento de enfermedades crónicas, como la diabetes, debe planificarse con mucha anticipación y no intentar brindar toda la información que se pretende compartir en una consulta o dos. Debido a los factores asociados con el duelo, relacionados con el conocimiento de que tiene de una enfermedad crónica incurable, se espera que el paciente (ya sea por rechazo o en estado de shock) no retenga mucha de la información proporcionada en el proceso. Por este motivo, entre otras cosas, conviene asegurarse de que en este punto el paciente se encuentre en compañía de un familiar o amigo cercano. Asimismo, en la consulta en la que se comunica el diagnóstico de una enfermedad crónica, es recomendable centrarse en el mejor y no en el peor pronóstico. Los malos escenarios deberían ir 
informándose en la medida en la que el paciente presente dificultades/renuencia a respetar las indicaciones médicas $(1,2,10)$.

Se debe dejar siempre tiempo en las consultas para responder las dudas que el paciente pueda tener. Es fundamental comprender que son infrecuentes los pacientes que comunican espontáneamente sus pareceres, dudas o preocupaciones, por lo que el médico tiene la obligación de hurgar en la mente del paciente en busca de sus dudas $(1,2,10)$.
Asimismo, se hace mandatorio que, durante las consultas, se indique al paciente la importancia de los controles. Para lograr este objetivo, suele ser de ayuda explicar al paciente cuáles son las acciones, evaluaciones o análisis que el médico tiene planeado para las siguientes consultas. Igualmente, conviene explicar por qué dichas actividades no pueden ser desarrolladas en una sola consulta $(10,11)$.

La tabla 3 presenta de manera sucinta las habilidades de comunicación esperadas en el personal de blanco, según el Consenso de Kalamazoo (12).

TABLA 3. HABILIDADES DE COMUNICACIÓN ESENCIALES: CONSENSO DE KALAMAZOO (12).

\begin{tabular}{|c|c|}
\hline Etapa de la consulta & \begin{tabular}{ll} 
& \multicolumn{1}{c}{ Habilidades esperadas } \\
- $\quad$ Permitir la apertura del paciente. \\
- $\quad$ Establecer/mantener conexión con el paciente.
\end{tabular} \\
\hline Obtener Información & $\begin{array}{l}\text { - } \quad \text { Usar preguntas abiertas y cerradas adecuadamente. } \\
\text { - } \quad \text { Estructurar, clarificar y resumir la información. } \\
\text { - Escucha activa usando técnicas no verbales (por ejemplo, contacto ocular) y } \\
\text { verbales (por ejemplo, palabras de ánimo). }\end{array}$ \\
\hline $\begin{array}{l}\text { Comprender la } \\
\text { perspectiva del } \\
\text { paciente }\end{array}$ & $\begin{array}{l}\text { - } \quad \text { Explorar factores contextúales (por ejemplo, familia, cultura, género, edad, } \\
\text { estado socioeconómico, espiritualidad). } \\
\text { - } \quad \text { explorar creencias, preocupaciones y expectativas sobra la salud y la } \\
\text { enfermedad. } \\
\text { - Conocer y responder a las ideas, sentimientos y valores de los pacientes. }\end{array}$ \\
\hline Compartir información & $\begin{array}{l}\text { - } \quad \text { Utilizar lenguaje que el paciente pueda comprender. } \\
\text { - } \quad \text { Comprobar que nos entienda. } \\
\text { - } \quad \text { Animar a que realice preguntas. }\end{array}$ \\
\hline $\begin{array}{l}\text { Alcanzar un acuerdo } \\
\text { sobre problemas y } \\
\text { planes }\end{array}$ & $\begin{array}{l}\text { - } \quad \text { Animar al paciente a participar en decisiones hasta que él desee. } \\
\text { - } \quad \text { Comprobar la buena voluntad y habilidades del paciente para seguir el plan. } \\
\text { - } \quad \text { Identificar y enumerar recursos y apoyos. }\end{array}$ \\
\hline Cerrar la sesión & $\begin{array}{l}\text { - } \quad \text { Preguntar si el paciente tiene otras cuestiones o preocupaciones. } \\
\text { - } \quad \text { Resumir y afirmar el acuerdo con el plan de acción. } \\
\text { Discutir el seguimiento (por ejemplo, siguiente consulta, plan para resultados } \\
\text { inesperados). }\end{array}$ \\
\hline
\end{tabular}

\section{Construyendo costumbres terapéuticas}

La especie humana es una de costumbres, y esto se debe en gran parte a la inclinación que tiene el cerebro por economizar la poca energía que tiene disponible para controlar todo el cuerpo, buscando constantemente estrategias (bajo la ley del menor esfuerzo) para llevar a cabo sus tareas. Este es uno de los motivos por los cuales, eventualmente, transforma procesos nuevos en procesos aprendidos y automáticos, con el objeto de dedicar menor atención a la realización de los mismos (13).

Anteriormente se consideraba que el tiempo necesario para la implementación de una nueva rutina requería de 21 días de constancia. Lamentablemente, nuevos estudios mueven la marca a los 76 días. En otras palabras, para que una función nueva pueda ser transferida desde las áreas cerebrales que requieren de procesos como la atención y la motivación como motores ejecutores, a otras donde solo se requiere la costumbre para hacerlas, implica suma constancia en dicha práctica. Y esto es de fundamental importancia que lo comprenda el personal de blanco que tiene a su cargo el tratamiento de un paciente con una patología crónica, ya que deberá establecer estrategias que garanticen la ejecución de las actividades que integran el nuevo estilo de vida del paciente (14).

La tabla 4 describe algunas estrategias que pueden ayudar a que el paciente con diabetes se adecue a su nuevo estilo de vida, posterior al diagnóstico $(15,16)$. 
TABLA 4. ESTRATEGIAS QUE AYUDAN A ALCANZAR OBJETIVOS COMPLEJOS $(15,16)$.

\begin{tabular}{|c|c|}
\hline Estrategia & Descripción \\
\hline $\begin{array}{l}\text { Transformar el } \\
\text { deseo en un } \\
\text { objetivo concreto }\end{array}$ & $\begin{array}{l}\text { Esto debe hacerse utilizando una herramienta conocida como el modelo MARTE (estas son } \\
\text { las siglas de las características que debe cumplir un objetivo para ser considerado como tal } \\
\text { y no como una intención o un deseo). } \\
\text { MARTE significa que un objetivo debe ser Medible, Alcanzable, Retador, Tiempo (fecha de } \\
\text { inicio y fecha límite) y Específico. Es importante que el objetivo sea muy específico (no } \\
\text { general) y que sea alcanzable y desafiante (un equilibrio difícil, pero posible). }\end{array}$ \\
\hline $\begin{array}{l}\text { Dividir el objetivo } \\
\text { en micro-acciones }\end{array}$ & $\begin{array}{l}\text { A partir de este objetivo MARTE, se deben marcar pasos muy pequeños o medidas } \\
\text { específicas con las que se pueda lograr el objetivo a largo plazo. Las micro-acciones que se } \\
\text { ejecutan constantemente son esenciales para que el objetivo sea sostenible en el tiempo. } \\
\text { Por ejemplo, si se comienza una dieta muy estricta, se perderá peso muy rápidamente. Sin } \\
\text { embargo, se ha comprobado que, en la mayoría de los casos, la persona recupera peso al } \\
\text { cabo de un tiempo, porque no ha podido mantener esta dieta de forma sostenible. }\end{array}$ \\
\hline $\begin{array}{l}\text { Superar los micro- } \\
\text { fracasos }\end{array}$ & $\begin{array}{l}\text { Si se cumple el punto } 2 \text {, solo pueden ocurrir micro-fracasos, no mayores. Los micro- } \\
\text { fracasos son fáciles de superar porque son de poca importancia. En otras palabras, si un } \\
\text { día se fracasa, el mundo no se hundirá. Incluso si pasa lo mismos dos o tres días, siempre y } \\
\text { cuando se relativice su relevancia y el plan se siga cumpliendo. }\end{array}$ \\
\hline \multirow[t]{2}{*}{$\begin{array}{l}\text { Premiar los micro- } \\
\text { logros }\end{array}$} & $\begin{array}{l}\text { El cerebro, según numerosos estudios, funciona a base de recompensas. Si se van dando } \\
\text { pequeños premios durante el camino, cuando se vayan logrando pequeños avances, el } \\
\text { cerebro liberará dopamina y la persona se sentirá mucho más animada y motivada para } \\
\text { continuar avanzando hacia el objetivo. }\end{array}$ \\
\hline & $\begin{array}{l}\text { Estos premios deben ser sencillos, no deben representar un costo económico importante } \\
\text { y deben ser sanos. }\end{array}$ \\
\hline $\begin{array}{l}\text { Comprometerse } \\
\text { con alguien }\end{array}$ & $\begin{array}{l}\text { Está demostrado que si se comparte con alguien importante (la pareja, hijo, un amigo o el } \\
\text { tratante) el firme propósito de lograr un objetivo, es mucho más probable que se lo } \\
\text { obtenga, debido a que esa persona importante irá preguntando por el progreso. Una buena } \\
\text { relación médico - paciente es vital para lograr este compromiso. }\end{array}$ \\
\hline
\end{tabular}

\section{La motivación y la activación}

A esta altura resulta conveniente el análisis de la motivación, ya que dicho componente cognitivo ya estuvo siendo mencionado en párrafos anteriores. El concepto de motivación es esencial a la hora de explicar el porqué de la mayoría de las acciones y conductas que se llevan a cabo a lo largo de la vida $(17,18)$.

El término motivación proviene del latín movere que hace referencia a aquello que se mueve o tiene virtud para mover. Según esta definición, la motivación sería la inclinación de alguien a realizar o no realizar algo, por tanto, la conducta no ocurre de forma espontánea, sino que estaría inducida por estímulos o motivos internos o externos (incentivos o recompensas) $(17,18)$.

En última instancia, la motivación es el proceso psicológico que nos permite describir las fuerzas que actúan sobre un organismo o aquellas que se originan desde su interior, y que dan lugar a que una conducta se instaure, se mantenga, se oriente hacia una determinada dirección, con una determinada intensidad y frecuencia y que finalmente se detenga o termine. Asimismo, ello supone analizar todas las reacciones subjetivas que tienen lugar en el organismo durante el mencionado proceso $(17,19)$.

Las investigaciones sobre la motivación, a diferencia de otros procesos psicológicos como la atención, la percepción o el aprendizaje, fueron surgiendo bajo aproximaciones teóricas desde diferentes disciplinas, y no como resultado de la investigación experimental. A modo de resumen, podríamos establecer cinco grandes grupos de teorías: 1) biológicas; 2) de la personalidad; 3) conductuales; 4) cognitivas, y 5) emocionales. Evitando profundizar demasiado en el tema, el presente artículo se limita a proponer una teoría integradora de las anteriormente mencionadas (17-20).

Es importante recordar que la motivación es un proceso de ajuste que está estrechamente relacionado con la supervivencia y el crecimiento personal del individuo. Por tanto, para muchos autores, la motivación es un proceso dinámico, cuyo principal objetivo es aumentar la probabilidad de que el organismo se adapte a los cambios del entorno. En otras palabras, sería un elemento fundamental para que el individuo logre sus objetivos, ya sea para la supervivencia o para la consecución de otros fines $(18,19)$.

La activación es la cualidad que estimula la motivación 
y es responsable del inicio, mantenimiento, intensidad y finalización de la conducta motivada. Fue introducida a la psicología por un fisiólogo de la Universidad de Harvard, Walter B. Cannon (1872-1945), basado en su trabajo sobre las funciones psicofisiológicas de los estados emocionales y motivacionales. Desde un punto de vista fisiológico, el concepto de activación se refiere al aumento de la actividad fisiológica, que se relaciona principalmente con la actividad del sistema nervioso autónomo (SNA) (18).

Existe una relación entre activación y rendimiento. Se ha puesto de manifiesto que mientras la activación se relaciona de forma lineal con la actividad fisiológica, no ocurre lo mismo con el rendimiento. La eficacia en la ejecución de determinadas tareas depende del nivel de motivación (activación) y del nivel de dificultad de la tarea. El nivel de motivación tiene un punto óptimo entre los valores intermedios de activación, de forma que valores excesivamente altos y bajos empeoran el rendimiento en la realización de una tarea $(18,19,21)$. Por otro lado, respecto a la relación entre activación y dificultad de la tarea, autores han defendido que el nivel óptimo de motivación era más alto para las tareas más fáciles, mientras que sería más bajo para las más difíciles, pero sobre esto no existe unanimidad por la compleja relación entre las variables de activación y rendimiento. Por último, señalar que la activación es uno de los dos componentes básicos del proceso motivacional, y que es una condición necesaria, aunque no suficiente, para que se desencadene la conducta motivada. Lo fundamental del proceso motivacional sería en última instancia la dirección de la conducta, es decir, las condiciones que impulsan al organismo a pasar de una actividad a otra, ya que en definitiva el organismo siempre está en continua actividad $(18,19,21)$.

\section{De la dirección de la conducta}

Si bien el concepto de activación puede verse desde una perspectiva cuantitativa, la dirección podría explicarse desde un enfoque cualitativo. El concepto de directividad de la conducta abarca tanto los objetos determinados genéticamente como los objetivos típicos de la conducta humana. De modo que comemos cuando tenemos hambre, descansamos cuando estamos agotados, o huimos cuando percibimos peligro. Por otro lado, tratamos de lograr metas académicas, emocionales, intelectuales, etcétera, en relación con otro tipo de motivos. Estos últimos ejemplos de metas y objetivos nos diferencian a los humanos de otros animales e incluso a los humanos unos de otros, en función de factores como el desarrollo evolutivo, las habilidades psicológicas, las variables de aprendizaje y la personalidad, aspectos que determinan y determinan la dirección de la conducta $(19,21)$.

Existen dos variables que intervienen en la consecución de los objetivos $(22,23)$ : a) La expectativa de alcanzar el objetivo propuesto depende de la percepción que tenga el individuo de la proximidad o lejanía del objetivo y determina el esfuerzo, la persistencia y el compromiso para lograrlo. Por ejemplo, los jugadores de un equipo de baloncesto seguirán motivados y luchando siempre que tengan la expectativa de poder superar al oponente en el marcador. Asimismo, un alumno no tiene que prepararse para un examen si no tiene la expectativa de poder aprobarlo.

b) El grado de atracción del objetivo marcado, que se da cuando una persona dirige su comportamiento hacia aquellos que le son atractivos. Un músico estudiará y practicará con su instrumento durante horas, según su atracción por la música y el instrumento con el que esté estudiando. Sin embargo, con el tiempo, las metas cambian y las motivaciones pueden tomar diferentes direcciones. Las personas evolucionan de manera diferente y las preferencias pueden variar, lo que indica nuevos objetivos. Además, la aparición de otras metas que sean más asequibles o atractivas y que requieran menos esfuerzo o compromiso puede orientar la motivación conductual en nuevas direcciones.

Cabe señalar que las posibilidades de selección de objetivos determinan de manera importante el componente direccional, por lo que cuando hay pocas opciones para seleccionar objetivos, es fácil decidir qué tipo de comportamiento se debe aplicar. Por el contrario, cuando hay muchas opciones, elegir entre múltiples objetivos aumenta la dificultad para tomar decisiones y las oportunidades para volver a cambiar de motivación, sobre todo si se está interesado en varios al mismo tiempo. Esto debe tenerse siempre en cuenta, ya que el tratante de un paciente con diabetes debe presentarle suficientes opciones dentro de su nuevo estilo de vida, pero no tantas que lo abrumen y dificulten la elección de los pasos que tomará para lograr una forma de vida más saludable (22-24).

En síntesis, la motivación juega un papel fundamental en la adherencia terapéutica. Es crucial que el tratante sepa lidiar con aquello que el paciente debe hacer y aquello que realmente puede lograr. Establecer objetivos terapéuticos demasiado elevados desde un principio llevarán a que el paciente termine abandonando el tratamiento independientemente a que ello vaya en detrimento de su salud (21). 
Emociones y salud

Los humanos tienen la tendencia a creerse racionales y a considerar a las emociones como la base de decisiones desacertadas. De ahí que, cuando se busca tomar una decisión importante, se hace el inhumano esfuerzo de eliminar todo indicio emocional de tal decisión. Paradójico resulta que son las emociones uno de los principales mecanismos que ha permitido a los seres vivos sobrevivir en la cadena evolutiva y alimentaria $(25,26)$.

Desde una perspectiva multidimensional, la emoción se ha definido como una respuesta biopsicosocial transitoria a eventos que tienen implicaciones para nuestro bienestar y pueden requerir una acción inmediata. Esta definición recoge la visión multinivel de las emociones en el plano biológico, psicológico y social $(27,28)$ : a) Las emociones son biológicas porque implican respuestas fisiológicas del sistema nervioso central y sistema nervioso autónomo.

b) Son psicológicas porque implican los procesos mentales específicos necesarios para la obtención y la regulación de la respuesta, las actividades mentales concretas y para incentivar el comportamiento.

c) Son sociales porque con frecuencia son provocadas por factores sociales y, a su vez, tienen un significado social concreto cuando se expresan y manifiestan.

Una emoción real como la felicidad, la tristeza, la vergüenza o la simpatía es una serie compleja de reacciones químicas y neuronales que forman un patrón único. Debido a esta propiedad de unicidad que tienen las emociones, no tiene sentido evaluar cada emoción y usarla para crear un modelo integrador. Sin embargo, existen ciertas características que son comunes a la mayoría de las emociones, que se exponen en la tabla $5(25,27-30)$.

\section{TABLA 5. CARACTERÍSTICAS COMUNES A TODAS LAS EMOCIONES.}

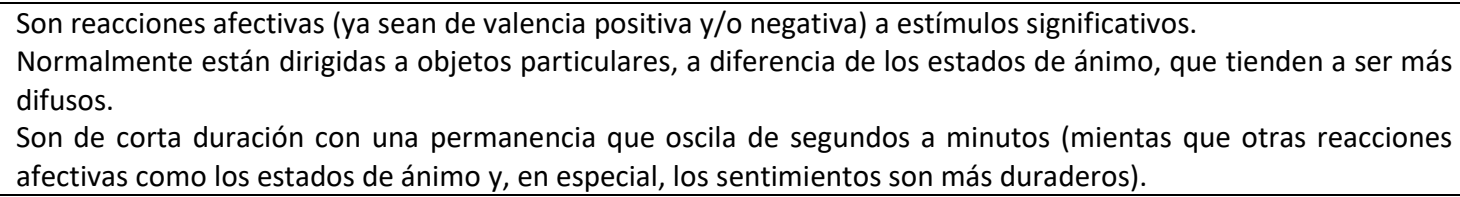

Existen muchas variables en las personas que reciben un mensaje que pueden influir en su procesamiento de la información y cambio de actitudes. Por ejemplo, la personalidad, el estilo cognitivo, su sistema de valores y creencias, etc. Las emociones del receptor de una noticia, pueden afectar a los juicios cognitivos y las evaluaciones a través de diferentes procesos psicológicos. En términos generales, las personas con estados de ánimo y emociones de valencia positiva suelen dedicar menos atención a pensar sobre los mensajes que reciben que las personas con estado de ánimo negativo $(27,28,30)$. Una consecuencia importante de esta diferencia en el procesamiento de la información es que en condiciones de alegría (frente a las de tristeza) se tiende a discriminar menos entre propuestas muy convincentes y poco convincentes. Es por ello que es de suma importancia la práctica de la comunicación asertiva ya que, si bien es virtualmente imposible generar un ambiente feliz para la comunicación de una mala noticia, al menos se debe evitar generar un ambiente aciago que comprometa la actitud del paciente para comprender lo que se le está comunicando $(27,28,30)$.

Dada la naturaleza crónica de la diabetes, es importante reiterar que la aparición de una enfermedad crónica en la vida de una persona siempre implica una situación de crisis ya que tiene grandes implicaciones físicas, psicológicas y sociales. Esta situación, por tanto, tiene grandes efectos en el paciente y una alteración de su comportamiento y su forma de vida habitual, lo que conduce a una situación de desequilibrio que, en última instancia, puede describirse como una situación estresante. Por otro lado, la enfermedad no solo genera estrés en la persona que la padece, sino que también tiene efectos más o menos estresantes en los familiares del paciente. La dinámica de las relaciones personales dentro de la familia está influenciada por la enfermedad (y en ocasiones la hospitalización), lo que provoca cambios en la representación de los roles habituales, lo que en sí mismo es una fuente de estrés que agrava la situación del paciente $(1,3,6)$.

\section{La adherencia terapéutica}

Desde una perspectiva global, vivimos en una sociedad que envejece. La esperanza de vida está aumentando, lo que hace que los problemas de salud crónicos sean más relevantes y frecuentes. Ante esta situación, actualmente existen procedimientos de diagnóstico y una variedad de tratamientos altamente efectivos para determinar qué enfermedad estamos enfrentando y cómo tratarla y superarla. Sin embargo, esta 
efectividad se ve mermada porque un porcentaje muy elevado de pacientes no se adhiere adecuadamente a las pautas terapéuticas prescritas $(31,32)$.

Dado que no parece razonable creer que el tratamiento solo funcionará porque ha sido prescrito, cualquier profesional de la salud considera que la falta de cumplimiento es un problema obvio y su resolución sigue siendo uno de los principales objetivos de salud. Uno de los países con mayor adherencia terapéutica es Estados Unidos de América y, sin embargo, apenas llega al $51 \%$. En países en desarrollo como los de América Latina, este porcentaje está por debajo del 25 $\%$. Si solo nos centramos en la medicación como método terapéutico, hay estudios que demuestran que a los 6 meses entre un $30 \%$ y un $80 \%$ de los pacientes dejan de tomar su medicación (33-35).

Entonces, ¿qué es la adherencia terapéutica? Primero, necesitamos distinguirla del cumplimiento terapéutico. Este último se refiere al grado en que el paciente se adhiere al tratamiento prescrito, lo que está más relacionado con una relación paternalista entre médico y paciente. Por otro lado, la adherencia terapéutica se relaciona con un modelo más recíproco de la relación médico-paciente, ya que se define como la participación activa y la colaboración voluntaria del paciente en una conducta mutuamente aceptada para lograr el resultado terapéutico deseado $(33,36)$.

En las investigaciones sobre la adherencia terapéutica se han estudiado un conjunto amplio de factores explicativos de la adherencia o falta de adherencia, que se contemplan en una u otra teoría. Un primer grupo de factores, que está claramente relacionado con la adherencia terapéutica, es el constituido por las peculiaridades del propio régimen terapéutico. Los tratamientos médicos tienen características muy diferentes: complejidad, duración, coste, efectos secundarios y grado en que exigen modificación de los hábitos de vida del paciente $(34,35)$. A mayores modificaciones de ese estilo de vida, más difícil se hace la adherencia terapéutica.

El segundo grupo de factores está relacionado con las características de la enfermedad. En particular, la gravedad de la enfermedad percibida por el paciente y la vulnerabilidad percibida a la enfermedad o a sus consecuencias $(34,35)$. Esa percepción viene ligada al tercer grupo de factores, que son las características psicosociales del paciente. Aquí se destaca que la observancia del tratamiento viene determinada por factores cognitivos y emocionales (conocimientos, creencias, actitudes, estados de ánimo, características de personalidad, motivación, entre otros). Los factores psicosociales se relacionan intrínsicamente con un cuarto grupo de factores, que son los relativos al contexto sociocultural del paciente. Por último, uno de los factores más relevantes para el cumplimiento de los tratamientos médicos es la interacción entre el médico y el paciente, la cual se basa en gran medida en una buena comunicación, esencial para que el paciente se adhiera el tratamiento (34-36).

\section{Gestionando la adherencia terapéutica}

Para gestionar la adherencia terapéutica (sobre todo para reducir las tasas de incumplimiento o falta de adhesión), se deben abordar dos ámbitos: el de la conducta del paciente, y el de la conducta del profesional sanitario (37-39). Las tablas 6 y 7 resumen lo que se puede hace en cada uno de estos ámbitos.

TABLA 6. GESTIONANDO LA CONDUCTA DEL PACIENTE.

Aquí el profesional sanitario debe:

- Incrementar la probabilidad de que el paciente acuda a la consulta.

- Hacer que comprenda adecuadamente las prescripciones terapéuticas.

- Reducir los aspectos aversivos de la prescripción.

- Detectar a los pacientes que puedan tener bajo nivel de adhesión.

- Adaptar la prescripción al estilo de vida del paciente.

- Utilizar refuerzos tangibles y, cuando sea posible, contratos conductuales.

- Enseñar estrategias para implantar el autocontrol del paciente mediante programas de refuerzo.

TABLA 7. GESTIONANDO LA CONDUCTA DEL PROFESIONAL SANITARIO.

Por lo que se refiere al profesional sanitario, lo más importante es mejorar su estilo de relación (y de comunicación) con el paciente, crucial para el éxito de la adherencia terapéutica. Lo anterior se puede lograr:

- Simplificando las instrucciones, a través del uso de un lenguaje claro.

- Usando oraciones específicas y concretas.

- Organizando la información y dividiendo los tratamientos complicados en partes más breves y fáciles.

- Resaltando la información relevante, explicando qué es importante y por qué, y presentándola al comienzo de la consulta.

- Utilizando instrucciones escritas.

- Verificando la comprensión del paciente (pidiéndole que repita las instrucciones o que explique la información recibida en sus propias palabras). 


\section{CONCLUSIÓN}

En conclusión, cabe resumir que se ha demostrado que el "cumplimiento" o "adherencia" al régimen terapéutico aumenta significativamente cuando la satisfacción del paciente se incrementa con el trato amable y cálido con su médico, con consultas o sesiones informativas, y cuando se le da retroalimentación.

Asimismo, la satisfacción mutua (de paciente y médico) con la relación terapéutica aumenta la calidad de la atención y los resultados de los tratamientos. Facilitar la comunicación de los profesionales con los pacientes y sus familiares facilita su implicación en el tratamiento y cuidado del paciente, y permite controlar las dificultades que puedan surgir en la aplicación del programa terapéutico. Finalmente, la mejora de las relaciones interpersonales de los usuarios con los profesionales de la salud ayuda a incrementar las habilidades interpersonales de estos profesionales, así como su satisfacción laboral. Por tanto, tiene un impacto significativo en la calidad de la atención $(35,36)$.

\section{REFERENCIAS}

1. Hernández Ruiz de Eguilaz $M$, Batlle MA, Martínez de Morentin B, San-Cristóbal R, Pérez-Díez S, Navas-Carretero $S$, et al. Cambios alimentarios y de estilo de vida como estrategia en la prevención del síndrome metabólico y la diabetes mellitus tipo 2: hitos y perspectivas. Anales Sis San Navarra. 2016;39(2):269-289. URL.

2. Gómez-López VM, García-Ruiz ME, Barrientos-Guerrero C. Diabetes Mellitus: Aptitud clínica del médico de atención primaria. Educ. méd. 2006;9(1):35-39. URL.

3. Peralta G, Figuerola D. Aspectos psicosociales de la diabetes. Endocrinol Nutr. 2003;50(7):280-285. https://doi.org/10.1016/S1575-0922(03)74539-2

4. Ghaemi SN. The rise and fall of the biopsychosocial model. $\mathrm{Br} J$ Psychiatry. 2009;195(1):3-4. https://doi.org/10.1192/bjp.bp.109.063859

5. Rostosky SS, Travis, CB. Menopause research and the dominance of the biomedical model 1984-1994. Psychol. Women Q. 1996;20(2):285-312. https://doi.org/10.1111/j.1471-6402.1996.tb00471.x

6. Roos S. The Kubler-Ross Model: An Esteemed Relic. Gestalt Review. 2012;16(3): 312-315. https://doi.org/10.5325/gestaltreview.16.3.0312

7. Santrock JW. A topical approach to life-span development. Nueva York: McGraw-Hill; 2007.

8. Silva Aycaguer LC. Ciencia y pseudociencia: una distinción crucial. Rev Cubana Invest Bioméd. 1997;16(2):78-82. URL.

9. Simpson M, Buckman R, Stewart M, Maguire P, Lipkin M, Novack $D$, et al. Doctor-patient communication: the Toronto consensus statement. BMJ. 1991;303(6814):13851387. https://doi.org/10.1136/bmj.303.6814.1385

10. Blonde L. Current antihyperglycemic treatment guidelines and algorithms for patients with type 2 diabetes mellitus. Am J Med. 2010;123(3 Suppl):S12-S18. https://doi.org/10.1016/i.amimed.2009.12.005
Por último, si queremos asegurar la adherencia al tratamiento, y el éxito terapéutico, son necesarias unas condiciones de trabajo con un entorno y un tiempo mínimo para atender al paciente, que muchas veces, en varios hospitales del mundo, no es el ideal. El acto médico requiere el tiempo suficiente para tomar decisiones, brindar al paciente las explicaciones adecuadas, responder a sus dudas, confirmar que se han entendido las instrucciones y explicaciones y, en definitiva, comunicarse adecuadamente con cada paciente. Cuando este no es el caso, surgen tensiones entre el médico y el paciente, surgen dudas, sospechas, ambigüedad y abandono, y falla la relación médicopaciente, que es la causa central de problemas relacionados con el fracaso o éxito de la terapia. Estos son factores que se deben vencer, inculcando su estudio y abordaje ya desde la Universidad, durante la formación del estudiante en médico (37).

11. Mogensen CE. New treatment guidelines for a patient with diabetes and hypertension. J Hypertens Suppl. 2003;21(1):S25-S30. URL.

12. Makoul G. Essential elements of communication in medical encounters: the Kalamazoo consensus statement. Acad Med. 2001;76(4):390-393 https://doi.org/10.1097/00001888-200104000-00021

13. Manzanero AL. Procesos automáticos y controlados de memoria: Modelo Asociativo (HAM) vs. Sistema de Procesamiento General Abstracto. Revista de Psicología General y Aplicada 2006;59(3):373-412. URL.

14. Brandstätter V, Lengfelder A, Gollwitzer PM. Implementation intentions and efficient action initiation. J Pers Soc Psychol. 2001;81(5):946-60. https://doi.org/10.1037//0022-3514.81.5.946

15. Hoque E. An Introduction to the Second Language Acquisition [Internet]. ResearchGate. 2017 [citado el 8 de febrero de 2021]. Disponible en: https://www.researchgate.net/publication/335690866 An Introduction to the Second Language Acquisition

16. Nicholls D, Sweet L, Campbell Westerway S, Gibbins A. The key to using a learning or skill acquisition plan. Australas J Ultrasound Med. 2014;17(4):141-145. https://doi.org/10.1002/j.2205-0140.2014.tb00235.x

17. Lotfizadeh AD, Edwards TL, Redner R, Poling A. Motivating operations affect stimulus control: a largely overlooked phenomenon in discrimination learning. Behav Anal. 2012;35(1):89-100. https://doi.org/10.1007/BF03392268

18. Moore J. On Psychological Terms That Appeal to the Mental. Behavior and Philosophy 2001;19:167-186. URL.

19. Barberá E. Marco conceptual e investigación de la motivación humana. Revista Española de Motivación y Emoción 2000;1:23-36.

20. Bernard LC, Mills M, Swenson L, Walsh RP. An evolutionary theory of human motivation. Genet Soc Gen Psychol Monogr. 2005;131(2):129-184. https://doi.org/10.3200/MONO.131.2.129-184 
21. Anaya-Durand A, Anaya-Huertas C. ¿Motivar para aprobar o para aprender? Estrategias de motivación del aprendizaje para los estudiantes. Tecnol. Ciencia Ed. (IMIQ).2010;25(1):5-14. URL.

22. Rudy D, Sheldon KM, Li Y, Kamble S, Bi X, Palermo F. Who Chooses Best? Explaining the Interactive Effect of Culture and Decision Maker on Children's Intrinsic Motivation. J. Cross-Cult. Psychol. 2015;46(4):471-488. https://doi.org/10.1177/0022022115572684

23. Díaz Méndez D, Latorre Postigo JM. Psicología Médica. Barcelona: Elsevier; 2015.

24. de Jesus SN, Lens W. An Integrated Model for the Study of Teacher Motivation. Appl. Psychol. 2005;54(1):119-134. https://doi.org/10.1111/j.1464-0597.2005.00199.x

25. LeDoux JE. Emotional memory systems in the brain. Behav Brain Res. 1993;58(1-2):69-79. https://doi.org/10.1016/0166-4328(93)90091-4

26. Dalgleish T. The emotional brain. Nat Rev Neurosci. 2004;5(7):583-589. https://doi.org/10.1038/nrn1432

27. Hall JA, Matsumoto D. Gender differences in judgments of multiple emotions from facial expressions. Emotion. 2004;4(2):201-206. https://doi.org/10.1037/15283542.4.2.201

28. Kitayama S, Markus HR, Matsumoto H. Culture, self, and emotion: A cultural perspective on "self-conscious" emotions. In: Tangney JP, Fischer KW, eds. Self-conscious emotions: The psychology of shame, guilt, embarrassment, and pride. New York: Guilford Press; 1995. pp. 439-464.

29. Damasio A. (2011) Neural basis of emotions. Scholarpedia 2011;6(3):1804.

https://doi.org/10.4249/scholarpedia.1804

30. Rodrigues SM, Schafe GE, LeDoux JE. Molecular mechanisms underlying emotional learning and memory in the lateral amygdala. Neuron. 2004;44(1):75-91. https://doi.org/10.1016/j.neuron.2004.09.014

31. Turnheim K. When drug therapy gets old: pharmacokinetics and pharmacodynamics in the elderly. Exp Gerontol. 2003;38(8):843-853. https://doi.org/10.1016/s05315565(03)00133-5

32. Tragaki A. The New World Looks Younger as the Old World Gets Older: The Fertility Divide between The US and Europe. European View 2008:7(2):247-254. https://doi.org/10.1007/s12290-008-0054-6

33. Ginarte Arias Y. La adherencia terapéutica. Rev Cubana Med Gen Integr. 2001;17(5): 502-505. URL.

34. Libertad MA. Repercusiones para la salud pública de la adherencia terapéutica deficiente. Rev Cubana Salud Pública. 2006; 32(3). URL.

35. Ortiz P Manuel, Ortiz P Eugenia. Health psychology: A key to understand therapeutic adherence. Rev. méd. Chile. 2007;135(5):647-652. https://doi.org/10.4067/S003498872007000500014

36. Conthe $P$ Márquez Contreras $E$, Aliaga Pérez A, Barragán García B, Fernández de Cano Martín MN, González Jurado $M$, et al. Adherencia terapéutica en la enfermedad crónica: estado de la situación y perspectiva de futuro. Revista Clínica Española. 2014;214(6):336-344. URL.

37. World Health Organization. Adherence to Long-term Therapies [Internet]. World Health Organization. 2003 [citado el 8 de febrero de 2021]. Disponible en: http://www.who.int/chp/knowledge/publications/adhere nce report/en/

38. Orueta R, Toledano P, Gómez-Calcerrada RM. Actualización en Medicina de Familia: Cumplimiento terapéutico. SEMERGEN-Medicina de Familia. 2008;34(5):235-243. https://doi.org/10.1016/S1138-3593(08)71889-6
39. Bertoldo P, Ascar G, Campana Y, Martín T, Moretti M, Tiscornia L. Cumplimiento terapéutico en pacientes con enfermedades crónicas. Rev Cubana Farm. 2013;47(4):468474. URL. 